Pacific Journal of Mathematics

STRONG HEREDITY IN RADICAL CLASSES 


\title{
STRONG HEREDITY IN RADICAL CLASSES
}

\author{
R. L. TANGEMAN
}

In a recent paper, $W$. G. Leavitt has called a radical class $\mathscr{P}$ in a universal class $\mathscr{W}$ of not necessarily associative rings strongly hereditary if $\mathscr{P}(I)=I \cap \mathscr{P}(R)$ for all ideals $I$ of any ring $R \in \mathscr{W}$. In this paper, strongly hereditary radicals are investigated and a new construction is provided for the minimal strongly hereditary radical containing a given class in $\mathscr{W}$. Nonassociative versions of some results of $E . P$. Armendariz on semisimple classes are proved, including a characterization of semisimple classes corresponding to strongly hereditary radicals.

Unless otherwise indicated, $\mathscr{W}$ is assumed to be a universal class of not necessarily associative rings. If $\mathscr{P}$ is any radical class in $\mathscr{W}$, we denote the class of $\mathscr{P}$-semisimple rings in $\mathscr{W}$ by $\mathscr{S} \mathscr{P}$. We use the notation $I \leqq R$ to denote that $I$ is an ideal of $R$. For any class $\mathscr{l l}$ we denote by $\mathscr{H} \mathscr{C l}$ and $\mathscr{F} \mathscr{C l}$, respectively, the homomorphic closure and ideal closure of $\mathscr{L}$.

For any radical class $\mathscr{P} \subseteq \mathscr{W}$, Leavitt in [7] has defined $\mathscr{G} \mathscr{P}=$ $\left\{J^{\prime} \mid J \leqq I \leqq R, J \in \mathscr{P}\right.$, and $J^{\prime}$ is the ideal of $R$ generated by $\left.J\right\}$. Radical classes $\mathscr{P}$ for which $\mathscr{P}=\mathscr{G} \mathscr{P}$ are said to satisfy property (a). Theorem 1 of [7] states that a hereditary radical class $\mathscr{P}$ is strongly hereditary if and only if $\mathscr{P}$ satisfies property (a). In [8], it is shown that any subclass $\mathscr{C}$ of $\mathscr{W}$ is contained in a unique minimal radical class satisfying property (a).

Some preliminary results are required.

Lemma 1.1. [2]. Let $\mathscr{P}$ be any radical class in $\mathscr{W}$. Then $\mathscr{S} \mathscr{P}$ is hereditary if and only if for each $R \in \mathscr{V}$ with $I \leqq R$ we have $\mathscr{P}(I) \subseteq(R)$.

Lemma 1.2. Let $\mathscr{P}$ be any radical class. Then $\mathscr{P}$ is strongly hereditary if and only if both $\mathscr{P}$ and $\mathscr{S} \mathscr{P}$ are hereditary.

Proof. If $\mathscr{P}$ is strongly hereditary, $\mathscr{P}(I)=I \cap \mathscr{P}(R)$ for each $I \leqq R$, so $\mathscr{P}$ and $\mathscr{S} \mathscr{P}$ are hereditary. Suppose $\mathscr{P}$ and $\mathscr{S} \mathscr{P}$ are hereditary and let $I \leqq R$. By Lemma 1.1 $\mathscr{P}(I) \cong I \cap \mathscr{P}(R)$. Also since $\mathscr{P}(R) \in \mathscr{P}$ and $\mathscr{P}$ is hereditary, $I \cap \mathscr{P}(R) \in \mathscr{P}$. Since $I \cap \mathscr{P}(R) \leqq I$, we have $I \cap \mathscr{P}(R) \subseteq \mathscr{P}(I)$.

Lemma 1.3. Let $\mathscr{P}$ be a radical class satisfying property (a). Then $\mathscr{S} \mathscr{P}$ is hereditary. If $\mathscr{P}$ is hereditary, $\mathscr{P}$ satisfies property 
(a) if and only if $\mathscr{S} \mathscr{P}$ is hereditary.

Proof. Suppose $\mathscr{P}$ has property (a). Let $J \leqq I \leqq R$ where $J \epsilon$ $\mathscr{P}$. Then $J^{\prime}$, the ideal of $R$ generated by $J$, belongs to $\mathscr{P}$, so $J^{\prime} \cong$ $\mathscr{P}(R)$. Thus $J \subseteq \mathscr{P}(R)$ and $\mathscr{P}(R)$ contains all the $\mathscr{P}$-ideals of $I$, so $\mathscr{P}(I) \subseteq \mathscr{P}(R)$. By Lemma 1.1, this means $\mathscr{S} \mathscr{P}$ is hereditary. If $\mathscr{P}$ and $\mathscr{S} \mathscr{P}$ are hereditary, then $\mathscr{P}$ is strongly hereditary by Lemma 1.2 , so $\mathscr{P}^{2}$ satisfies property (a) by Theorem 1 of [7].

The semisimple class $\mathscr{S} \mathscr{P}$ may be hereditary even when $\mathscr{P}$ does not satisfy property (a). To see this, let $R$ be the ring generated over $G F(2)$ by the nonassociative symbols $\{x, y, z\}$, subject to the relations $x^{2}=x y=y x=x z=x, y z=z y=z x=y, z^{2}=z, y^{2}=0$. Then the only proper nonzero ideal of $R$ is $I=\{0, x, y, x+y\}$ and the only proper ideal of $I$ is $J=\{0, x\} . \quad R / I$ and $J$ are isomorphic simple idempotent rings, and $I / J$ is simple and nilpotent. Let $\mathscr{W}=\{0, R, J, I, I / J\}$ and $\mathscr{P}=\{0, R, J\}$. Then $\mathscr{P} \neq \mathscr{G} \mathscr{P}$ but $\mathscr{S} \mathscr{P}=\{0, I / J\}$ is hereditary.

2. Radical classes. In Theorem 2 of [2], it is shown that if $\mathscr{P}$ is a radical class in an alternative class $\mathscr{W}$, and if $R \in \mathscr{W}$ with $I \leqq$ $R$, then $\mathscr{P}(I) \leqq R$. The following theorem shows that radicals satisfying property (a) in an arbitrary universal class have the same property.

Theorem 2.1. Let $\mathscr{P}$ be any radical class in $\mathscr{W}$. The $\mathscr{P}$ satisfies property (a) if and only if for each $R \in \mathscr{W}, I \leqq R$ implies $\mathscr{P}(I) \leqq$ $R$.

Proof. Suppose $I \leqq R$ implies $\mathscr{P}(I) \leqq R$. Then $\mathscr{P}(I) \leqq \mathscr{P}(R)$ so $\mathscr{S} \mathscr{P}$ is hereditary by Lemma 1.1. If $\mathscr{P}$ does not have property (a), we have some $J \leqq I \leqq R$ with $J \in \mathscr{P}$ and $J^{\prime} \notin \mathscr{P}$, where $J^{\prime}$ is the ideal of $R$ generated by $J$. This means $\mathscr{P}\left(J^{\prime}\right) \neq J^{\prime}$. Since $\mathscr{S} \mathscr{P}$ is hereditary and $J \leqq J^{\prime}$, we have $\mathscr{P}(J)=J \subseteq \mathscr{P}\left(J^{\prime}\right)$. Since $\mathscr{P}\left(J^{\prime}\right) \leqq R$, we have $J$ contained in an ideal of $R$ properly smaller than $J^{\prime}$, contradicting the definition of $J^{\prime}$.

Conversely, suppose $\mathscr{P}$ has property (a) and let $I \leqq R$. Then $\mathscr{P}(I) \leqq I \leqq R$, so $\mathscr{P}(I)^{\prime} \in \mathscr{P}$ and from $\mathscr{P}(I)^{\prime} \leqq I$ if follows that $\mathscr{P}(I)^{\prime} \subseteq \mathscr{P}(I)$. Thus $\mathscr{P}(I)=\mathscr{P}\left(I^{\prime}\right) \leqq R$.

In [3] (Lemma 5) a result is proved which may be restated for our purposes as

Lemma 2.2. If $\mathscr{P}$ is a radical class in an alternative class, then $\mathscr{P}$ satisfies property (a).

This lemma, when applied with Theorem 2.1, shows that in alter- 
native classes $I \leqq R$ implies $\mathscr{P}(I) \leqq R$, thus providing another proof of Theorem 2 of [2].

We next note that property (a) may be satisfied by possibly nonradical classes of rings. For an example of this, let $\mathscr{C l}$ be the class of nilpotent rings in the universal class of all associative rings. Then if $J \leqq I \leqq R$ with $J \in \mathscr{l}$, we have $\left(J^{\prime}\right)^{3} \leqq J$ so that $J^{\prime} \in \mathscr{C}$. The following lemma shows that such classes, if also homomorphically closed, are only one step removed from being radical.

Lemma 2.3. If $\mathscr{C l}$ is homomorphically closed and satisfies property (a), then $\mathscr{L} \mathscr{C}=\mathscr{K}_{2}$ in the lower radical construction. (For details of this construction see [5]).

Proof. Let $R \in \mathscr{A}_{3}$ and let $R_{1}$ be an arbitrary homomorphic image of $R$. Then $R_{1}$ has an ideal $I \in \mathscr{C}_{2}$ and $I$ has a nonzero ideal $J \in \mathscr{C}$. Since $\mathscr{C l}$ satisfies property (a), $J^{\prime}$, the ideal of $R_{1}$ generated by $J$, is in $\mathscr{l}$. Thus each image of $R$ has a nonzero ideal in $\mathscr{l}$, which means $\mathscr{A}_{3} \subseteq \mathscr{H}_{2}$ so $\mathscr{L} \mathscr{M}=\mathscr{H}_{2}$.

Using Lemma 2.3 , we next prove that property (a) is preserved by passing to the lower radical.

THEOREM 2.4. If th is homomorphically closed and satisfies property (a), then $\mathscr{L} \mathscr{C l}$ satisfies property (a).

Proof. Let $\mathscr{P}=\mathscr{L} \mathscr{C}=\mathscr{C l}_{2}$, and suppose $\mathscr{P}$ does not have property (a). Then by Theorem 2.1 there is some $R$ with $I \leqq R$ but $\mathscr{F}(I)$ not an ideal of $R$. Since the union of a chain of $\mathscr{P}$-ideals of $R$ is again a $\mathscr{P}$-ideal of $R$ (see [1]), we may select by Zorn's Lemma a $\mathscr{P}$-ideal $F$ of $R$ which is maximal among those $\mathscr{P}$-ideals of $R$ which are contained in $\mathscr{P}(I)$ ( $F$ may of course be zero). We claim $\mathscr{P}(I) / F=\mathscr{P}(I / F)$. Clearly $\mathscr{P}(I) / F \cong \mathscr{P}(I / F)$. Let $K$ be the ideal of $I$ such that $K / F=\mathscr{P}(I / F)$. Then $\mathscr{P}(I) \cong K$ and $(K / F) /(\mathscr{P}(I) / F) \cong$ $K / \mathscr{P}(I) \in \mathscr{P}$, forcing $K \in \mathscr{P}$, which means $K \subseteq \mathscr{P}(I)$ so $\mathscr{P}(I) / F=$ $\mathscr{P}(I / F)$. Now if $R / F$ has a nonzero $\mathscr{P}$-ideal $P / F \subseteq \mathscr{P}(I) / F$, we would have $P \in \mathscr{P}$ violating the maximality of $F$.

Thus by passing if necessary to a homomorphic image, we may assume $\mathscr{P}(I) \subseteq I \leqq R$, and $\mathscr{P}(I)$ contains no nonzero $\mathscr{P}$-ideal of $R$. Since $\mathscr{P}(I) \in \mathscr{L}_{2}, \mathscr{P}(I)$ has a nonzero ideal $J_{1} \in \mathscr{L}$, and the ideal $J$ of $I$ generated by $J_{1}$ is also in $\mathscr{l l}$. Thus we have $J_{1} \subseteq J \subseteq \mathscr{P}(I) \subseteq$ $I \subseteq R$ where $J \in \mathscr{C}$ and $J \leqq I$. Also $J^{\prime}$, the ideal of $R$ generated by $J$, is contained in $I$, and $J^{\prime} \in \mathscr{C} \subseteq \mathscr{P}$. Thus $J^{\prime} \subseteq \mathscr{P}(I)$, contradicting the assumption that $\mathscr{P}(I)$ contains no nonzero $\mathscr{P}$-ideals of $R$.

The example following the proof of Lemma 1.3 may be used to show that in the nonassociative case the requirement in Theorem 2.4 
that $\mathscr{C}$ be homomorphically closed cannot be dropped. Let $\mathscr{W}$ be as in the example and $\mathscr{C}=\{R\}$. Then $\mathscr{C}$ has property (a) but $\mathscr{H} \mathscr{M}=\mathscr{P}$ does not.

It is shown in [8] that an arbitrary class is contained in a unique minimal radical class satisfying property (a) and in a unique minimal strongly hereditary radical class. The next few results provided countable construction which are at most one (Kuroš) step from these classes.

Theorem 2.5. Let $\mathscr{M} \subseteq \mathscr{W}$ with $\mathscr{W}$ any universal class. There exists a unique minimal class in $\mathscr{W}$ containing $\mathscr{H}$ which is homomorphically closed and has property (a).

Proof. Define $\mathscr{L}_{1}=\mathscr{C}$ and $\mathscr{L}_{n+1}=\mathscr{G} \mathscr{H} \mathscr{H}_{n}$ for each $n \geqq 1$. Then set $\mathscr{C}^{*}=\cup \mathscr{C}_{n}$, the union being taken over all positive integers $n$. $\mathscr{H}^{*}$ is easily seen to be homomorphically closed. Also $\mathscr{L}^{*}$ satisfies property (a), for if $J \leqq I \leqq R$ with $J \in \mathscr{C}^{*}$, then $J \in \mathscr{K}_{n}$ for some $n$ so that $J^{\prime} \in \mathscr{H}_{n+1} \subseteq \mathscr{H}^{*}$.

If $\mathscr{A}$ is any homomorphically closed class containing $\mathscr{A}$ and satisfying property (a), an easy induction shows $\mathscr{C}_{n} \subseteq \mathscr{A}$ for each $n$ so that $\mathscr{L}^{*} \subseteq \mathscr{A}$.

CoRollary [Leavitt]. For $\mathscr{l} \cong \mathscr{W}$ with $\mathscr{W}$ any universal class there is a unique minimal radical class in $\mathscr{W}$ containing $\mathscr{C l}$ which satisfies property (a).

Proof. This is immediate from Theorems 2.4 and 2.5.

Note that by Lemma 2.2 the radical $\mathscr{L} \mathscr{M}^{*}$ coincides with the lower radical $\mathscr{L} \mathscr{M}$ in alternative classes, and thus for such classes the above construction may be regarded as an alternate lower radical construction.

Theorem 2.6. Let $\mathscr{W}$ be a universal class $\mathscr{C} \subseteq \mathscr{W}$. There is a unique minimal class $\mathscr{L}^{\prime} \supseteqq \mathscr{l}$ which is homomorphically closed, hereditary, and satisfies property (a).

Proof. Define $\mathscr{C}_{1}=\mathscr{M}$ and for $n \geqq 1$ let $\mathscr{A}_{n+1}=\mathscr{G} \mathscr{F} \mathscr{H}\left(\mathscr{A}_{n}\right)$. Now set $\mathscr{C}^{\prime}=\cup \mathscr{C}_{n}$, the union being taken over all positive integers.

As in the proof of Theorem 2.5, $\mathscr{C}^{\prime}$ is homomorphically closed, hereditary, and has property (a). Also as before (induction) $\mathscr{C l}^{\prime} \subseteq$ $\mathscr{A}$ where $\mathscr{A}$ is any homomorphically closed hereditary class with property (a) containing $\mathscr{K}$. 
Corollary 2.7. If $\mathscr{L l}$ is any class, $\mathscr{L} \mathscr{M}^{\prime}$ is the unique minimal strongly hereditary radical class containing $\mathscr{L}$.

Proof. Since $\mathscr{M} \subseteq \mathscr{M}^{\prime}, \mathscr{M} \subseteq \mathscr{L} \mathscr{C}^{\prime}$. Since $\mathscr{L}^{\prime}$ is homomorphically closed, hereditary, and satisfies property (a), $\mathscr{L} \mathscr{M}^{\prime}$ has the same properties by Theorem 2.4 together with Theorem 2 of [6]. Now let $\mathscr{C} \subseteq \mathscr{P}$ where $\mathscr{P}$ is a strongly hereditary radical class. Then $\mathscr{P}$ is homomorphically closed and hereditary, hence satisfies property (a) by Lemmas 1.2 and 1.3. Hence by Theorem $2.6 \mathscr{C}^{\prime} \leqq \mathscr{P}$ and therefore $\mathscr{L} \mathscr{L}^{\prime} \subseteq \mathscr{P}$.

3. Semisimple classes. Using Theorem 2.1, nonassociative versions of certain theorems concerning semisimple classes can be given. In [4], semisimple classes of associative rings are characterized as those classes $\mathbb{Q}$ satisfying the following four properties:

(1) $Q$ is hereditary

(2) $Q$ is closed under subdirect sums

(3) $Q$ is extension closed

(4) If $I \leqq R$ and $0 \neq I / B \in \mathbb{Q}$ for some ideal $B$ of $I$, there is an ideal $A$ of $R$ with $A \subseteq I$ and $0 \neq I / A \in \mathbb{Q}$.

For possibly nonassociative classes, we have

THEOREM 3.1. $\mathscr{Q}$ is a semisimple class for a radical class $\mathscr{P}$ satisfying property (a) if and only if $\mathbb{Q}$ satisfies properties (1), (2), (3), and (4).

Proof. If $\mathscr{Q}=\mathscr{S} \mathscr{P}$ where $\mathscr{P}$ has property (a), then the proof of (1), (2), (3), and (4) go through as in the associative case (see [4]) using Theorem 2.1 and Lemma 1.3. Conversely, suppose $\mathbb{Q}$ satisfies (1), (2), (3), and (4). Then again as in the associative case $\mathscr{Q}$ is semisimple for some radical $\mathscr{P}$. Suppose $\mathscr{P}$ does not satisfy property (a). Then by Theorem 2.1 there is some $R$ with an ideal $I$ for which $\mathscr{P}(I)$ is not an ideal of $R$. Let $T$ be the ideal of $R$ generated by $P(I)$, then $\mathscr{P}(I) \leqq T \leqq I \leqq R$. Then by (1) and two applications of Lemma 1.1, $\mathscr{P}(\mathscr{P}(I))=\mathscr{P}(I) \subseteq \mathscr{P}(T) \subseteq \mathscr{P}(I)$ so $\mathscr{P}(I)=\mathscr{P}(T) \neq$ $T \leqq R$, and $T$ is the ideal of $R$ generated by $\mathscr{P}(T)$. Also $T / \mathscr{P}(T) \in$ $Q$ so by (4) there is an ideal $K$ of $R$ with $K \cong T$ so that $T / K$ is nonzero in $\mathscr{Q}$. Thus $K \geqq \mathscr{P}(T)$ so $K$ is an ideal of $R$ containing $\mathscr{P}(T)$, and $K$ is proper in $T$, a contradiction which proves the theorem.

In [4], an ideal $I$ of a ring $R$ is said to be large in $R$ if $I$ has nonzero intersection with every nonzero ideal of $R$. It is proved there that a radical class $\mathscr{P}$ in an associative universal class is hereditary if and only if $\mathscr{S} \mathscr{P}$ satisfies property $(\lambda)$ : If $I \leqq R$ with $I \in \mathscr{S} \mathscr{P}$ and $I$ large in $R$, then $R \in \mathscr{S} \mathscr{P}$. The same proof given there proves the 
following theorem, which is valid in an arbitrary universal class.

THEOREM 3.2. Let $\mathscr{P}$ be a radical class satisfying property (a). Then $\mathscr{P}$ is hereditary if and only if $\mathscr{S} \mathscr{P}$ satisfies property $(\lambda)$.

Theorem 3.1 and 3.2 may be combined to give the following charracterization of semisimple classes for strongly hereditary radicals:

THEOREM 3.3. $Q$ is a semisimple class for a strongly hereditary radical if and only if $\mathbb{Q}$ satisfies properties (1), (2), (3), (4), and $(\lambda)$.

Proof. Suppose $\mathscr{Q}=\mathscr{S} \mathscr{P}$ where $\mathscr{P}$ is strongly hereditary. Then $\mathscr{P}$ is hereditary and has property (a) so $\mathbb{Q}$ satisfies (1), (2), $(3),(4)$, and $(\lambda)$. Conversely, if $Q$ satisfies (1), (2), (3), (4), and $(\lambda)$, then $\mathscr{Q}=\mathscr{S} \mathscr{P}$ for a radical $\mathscr{P}$ satisfying property (a) by Theorem 3.1 and $\mathscr{P}$ is hereditary by Theorem 3.2. Thus $\mathscr{P}$ is strongly hereditary by Lemma 1.2 .

The following proposition and its corollary show that certain semisimple classes of associative rings satisfy property (a).

Proposition 3.4. If $\mathscr{C l}$ is a class of associative rings which is hereditary, extension closed, and contains all nilpotent associative rings, then Mll satisfies property (a).

Proof. Suppose $J \leqq I \leqq R$ where $J \in \mathscr{L}$, and let $J^{\prime}$ be the ideal of $R$ generated by $J$. Then $\left(J^{\prime}\right)^{3} \subseteq J$ so $\left(J^{\prime}\right)^{3} \in \mathscr{L}$. Also $J^{\prime} /\left(J^{\prime}\right)^{3} \in \mathscr{L}$, so $J^{\prime} \in \mathscr{C}$ since $\mathscr{C}$ is extension closed.

CoRollary 3.5. If $\mathscr{P}$ is a radical in an associative universal class such that $R^{2}=R$ for all $R \in \mathscr{P}$, then $\mathscr{S} \mathscr{P}$ has property (a).

Proof. $\mathscr{S P P}$ is easily verified to satisfy the hypotheses of Proposition 3.4 .

\section{REFERENCES}

1. S. A. Amitsur, Radicals in rings and bicategories, Amer. J. Math., 76 (1954), 100125.

2. T. Anderson, N. Divinsky, and A. Sulinski, Hereditary radicals in associative and alternative rings, Canad. J. Math., 17 (1965), 594-603.

3. - Lower Radical Properties for associative and alternative rings, J. London Math. Soc., 41 (1966), 417-424.

4. E. P. Armendariz, Closure properties in radical theory, Pacific J. Math., 26 (1968), $1-7$.

5. N. Divinsky, Rings and radicals, University of Toronto Press, Tronto, 1965. 
6. A. E. Hoffman and W. G. Leavitt, Properties inherited by the lower radical, Portugal. Math., 27 (1968), 63-66.

7. W. G. Leavitt, Strongly hereditary radicals, Proc. Amer. Soc., 21 (1969), 703-705.

8. - Radical and semisimple classes with specified properties, Proc. Amer.

Math. Soc., 24 (1970), 680-687.

Received April 12, 1971.

ARKANSAS State UNIVERSity 



\section{PACIFIC JOURNAL OF MATHEMATICS}

\section{EDITORS}

H. SAMELSON

Stanford University

Stanford, California 94305

C. R. HobBY

University of Washington

Seattle, Washington 98105
J. DUGUNDJI

Department of Mathematics

University of Southern California

Los Angeles, California 90007

RICHARD ARENS

University of California

Los Angeles, California 90024

\section{ASSOCIATE EDITORS}

E. F. BECKENBACH

B. H. NeumanN

F WoLF

K. YoshidA

\section{SUPPORTING INSTITUTIONS}

UNIVERSITY OF BRITISH COLUMBIA

UNIVERSITY OF SOUTHERN CALIFORNIA

CALIFORNIA INSTITUTE OF TECHNOLOGY

UNIVERSITY OF CALIFORNIA

MONTANA STATE UNIVERSITY

STANFORD UNIVERSITY

UNIVERSITY OF NEVADA

NEW MEXICO STATE UNIVERSITY

OREGON STATE UNIVERSITY

UNIVERSITY OF OREGON

OSAKA UNIVERSITY

UNIVERSITY OF TOKYO

UNIVERSITY OF UTAH

WASHINGTON STATE UNIVERSITY

UNIVERSITY OF WASHINGTON

$\stackrel{*}{*} \stackrel{*}{*} \stackrel{*}{*}{ }^{*}{ }^{*}$ MMEICAN MATHEMATICAL SOCIETY

NAVAL WEAPONS CENTER

Printed in Japan by International Academic Printing Co., Ltd., Tokyo, Japan 


\section{Pacific Journal of Mathematics}

\section{Vol. 42, No. $1 \quad$ January, 1972}

Tage Bai Andersen, On Banach space valued extensions from split faces ........

David Marion Arnold, A duality for quotient divisible abelian groups of finite

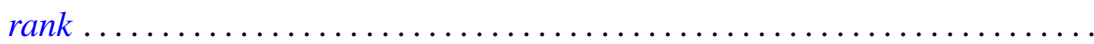

Donald Pollard Ballou, Shock sets for first order nonlinear hyperbolic



Leon Brown and Lowell J. Hansen, On the range sets of $H^{p}$ functions .........

Alexander Munro Davie and Arne Stray, Interpolation sets for analytic

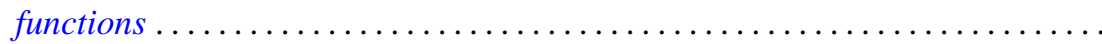

M. G. Deshpande, Structure of right subdirectly irreducible rings. II . . . . . . . . .

Barry J. Gardner, Some closure properties for torsion classes of abelian

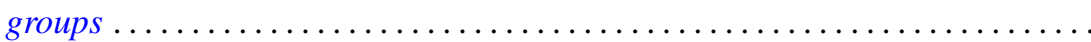

Paul Daniel Hill, Primary groups whose subgroups of smaller cardinality are

direct sums of cyclic groups . . . . . . . . . . . . . . . . . . .

Richard Allan Holzsager, When certain natural maps are equivalences .........

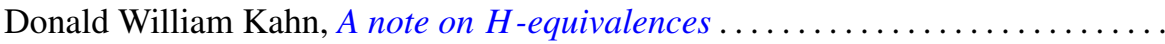

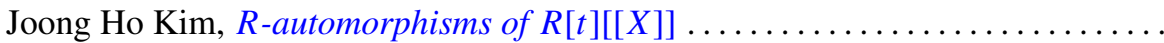

Shin'ichi Kinoshita, On elementary ideals of polyhedra in the 3-sphere.........

Andrew T. Kitchen, Watts cohomology and separability...

Vadim Komkov, A technique for the detection of oscillation of second order

ordinary differential equations .

Charles Philip Lanski and Susan Montgomery, Lie structure of prime rings of characteristic 2

Andrew Lenard, Some remarks on large Toeplitz determinants . .

Kathleen B. Levitz, A characterization of general Z.P.I.-rings. II .

Donald A. Lutz, On the reduction of rank of linear differential systems

David G. Mead, Determinantal ideals, identities, and the Wronskian ...

Arunava Mukherjea, A remark on Tonelli's theorem on integration in product

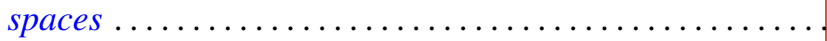

Hyo Chul Myung, A generalization of the prime radical in nonassociative rings.

John Piepenbrink, Rellich densities and an application to unconditionally nonoscillatory elliptic equations.

Michael J. Powers, Lefschetz fixed point theorems for a new class of multi-valued maps .

Aribindi Satyanarayan Rao, On the absolute matrix summability of a Fourier



T. S. Ravisankar, On Malcev algebras ......................... 227

William Henry Ruckle, Topologies on sequences spaces . . . . . . . . . . . . . 235

Robert C. Shock, Polynomial rings over finite dimensional rings . . . . . . . . . 251

Richard Tangeman, Strong heredity in radical classes . . . . . . . . . . . . . . 259

B. R. Wenner, Finite-dimensional properties of infinite-dimensional spaces . . . . 267 